\title{
Flexible best fit assembly of large aircraft components. Airbus A350 XWB case study
}

\author{
R. Arista, H. Falgarone \\ Airbus. 12, Rue Pasteur - 92150 Suresnes, France \\ rebeca.arista@airbus.com, hugo.falgaronedairbus.com
}

\begin{abstract}
The need for assembly parts and structures where the perfect fitting is not guaranteed due to manufacturing/assembly tolerances and/or the influence of several physical effects, i.e. gravity, is increasing constantly in different industrial sectors and in particular in the aerospace industry.

Some techniques have been developed to deal with it. In the field of large aero structures, custom-made parts using reverse engineering techniques are used to machine parts whose geometry requires to be customized for each produced aircraft. In the field of aircraft shells, a technique based on the characteristics of non-rigid components that can be slightly deformed to clear geometrical conditions by controlled forces to strain within its stress limits.

FITFLEX project exploit this last technique and was carried out by Airbus Group Innovations and Airbus. The objective is supporting the Airbus A350 XWB ramp-up and the current manual positioning process of the shell by force control, defining a measurement based assembly including a flexible best-fit system.

The case subject of study on FITFLEX project is the A350 XWB rear fuselage, a 14 meters by 5 meters side shell positioning process.
\end{abstract}

Keywords: flexible best-fit; assembly simulation; flexible assembly; tolerancing

\section{Introduction}

In the aerospace industry, large non-rigid and rigid components are assembled respecting defined functional requirements, such as geometric conditions or stress in the joints. This compliance to reach the final assembled part leads to non-value operations such as loads control, shimming, and rigging or ad-hoc parts manufacturing [1].

Non-rigid components can be slightly deformed to clear geometrical conditions by controlled forces to strain the part within its stress limits; these limits are more constrained for composite materials. This is the case of Airbus A350 XWB rear fuselage assembly, conformed by composite non-rigid shells held at the assembly station by an over constrained tooling to maintain its form.

Airbus A350 XWB benefits from being built with over 70 per cent advanced materials; combining carbon composites (53 per cent), titanium and modern aluminium

adfa, p. 1, 2011.

(C) Springer-Verlag Berlin Heidelberg 2011 
alloys, to create a lighter and more cost-efficient aircraft while also reducing maintenance requirements

Physical models generated during conceptual and design phase of the aircraft, to analyze the stress propagation during flight and define the assembly requirements could be reused to describe the part behavior of flexible parts during assembly.

FITFLEX project was carried out by Airbus Group Innovations and Airbus, with the objective of supporting A350 XWB ramp-up and the current manual positioning process of the shell by force control, defining a measurement based assembly including a flexible best-fit system.

The novelty of this approach is to re-use the physical model description made during the aircraft design phase, to adjust a real part during its assembly with no need of FEA computation or expertise, solving an optimization problem to find the best part positioning, that fulfills the requirements while controls and minimizes the constrains introduced to the part.

This paper is structured into six sections. Section 2 presents the background research which leads to the work made on FITFLEX project. Section 3 shows the project methodology and the contributions to the existing process. Section 4 presents the case study used to validate the development. Results obtained in this use case are described in Section 5. Finally, Section 6 presents the conclusions of the work. For confidentiality reasons of the use case subject of this paper, images and results information do not reflect the exact reality.

\section{Background research base of this work}

Research works performed in partnership between Airbus Group and ENS Cachan have been conducted for twenty years on several research topics, which are basis of the work performed in FITFLEX project. Best fit assembly considering behavior of components was studied using a helicopter door assembly use case [2]. Also, the assembly sequence influence on geometric deviations of parts was studied using a hydraulic system assembly case [3].

Research made in the frame of LOCOMACHS (Low Cost Manufacturing and Assembly of Composite and Hybrid Structures) EU project [4], on geometrical defects transfer in rigid and non-rigid components assemblies, lead to ANATOLE and ANATOLEFLEX software tools developments [5], which are used in FITFLEX project to define the use case assembly process and criteria. Also in LOCOMACHS framework, research on assembly process generation for composite structures [6], and measurement geometric characterization of flexible assembly [7] supported the project development.

Additional research made by Airbus Defence and Space on MISTRAL project [1], using reverse engineering techniques to manufacture customized parts for each aircraft to cope with discrepancies remaining after a rigid best-fit process, helped to analyze the scope and benefits of a flexible best fit process usage. 


\section{$3 \quad$ Methodology}

The methodology proposes considering only small component displacements and a linear system model; therefore we can rely on a linear static analysis to simulate forces and displacements, for each point of a discrete geometry [2].

In certain cases, linear behavior is not realistic enough, especially when there are clearances in the linkages and contact uncertainties. In these cases it is necessary to consider the assembly process as a nonlinear problem. Indeed, computing contact behavior involves using a nonlinear solver, and the use of finite element software is justified for the simulation. A prediction of gap between components is a case for which a non-linear model is needed [7][8].

Research found in the bibliography [9][10][11], consider parts contact during assembly to simulate and find the best assembly process, generating flexible assembly simulations with rigid and non-rigid parts by using a Finite Element Analysis (FEA) with computation tools (i.e. ABAQUS-SIMULIA by Dassault Systèmes).

In order to avoid the need of FEA computation and be able to have a quick and light computation on a shop floor tool, we approach the assembly process during the parts positioning stage, before the contact phase between the parts for its final assembly. Therefore, a reduced stiffness matrix of the part can be used as sensitivity matrix for computation of the optimal part positioning.

Thanks to the knowledge of the sensitivity matrix and assembly criteria, an optimization on the part positioning can be made within boundaries of the tolerance regions [12]. The next points describe the optimization problem solved using developed mathematical algorithms, how the sensitivity matrix is generated, and the process to follow in order to obtain the part optimal positioning, through a flexible best fit process for each aircraft.

\subsection{Linear system and optimization problem}

The mathematical method used relies on a linear system equation solving, using a sensitivity matrix that describes the system behavior. The equations are solved inside an optimization problem, to obtain the best setting of the component to fulfill the imposed characteristic requirements.

To describe the problem, we define the series of required characteristics as $C_{i}$, that need to be fulfilled within a given range during the assembly process, in order to reach the final assembled part functional requirement. These characteristics can be either displacements, on contact or non-contact points, or forces on contact points.

$$
C_{i} \in\left[C_{i, \min }, C_{i, \max }\right] \forall i=(1, \infty)
$$

We define positioning contact points as $D_{j}$, in which adjustable imposed displacements can be made within limits (small displacement assumption).

$$
D_{j} \in\left[D_{j, \min }, D_{j, \max }\right] \forall j=(1, \infty)
$$




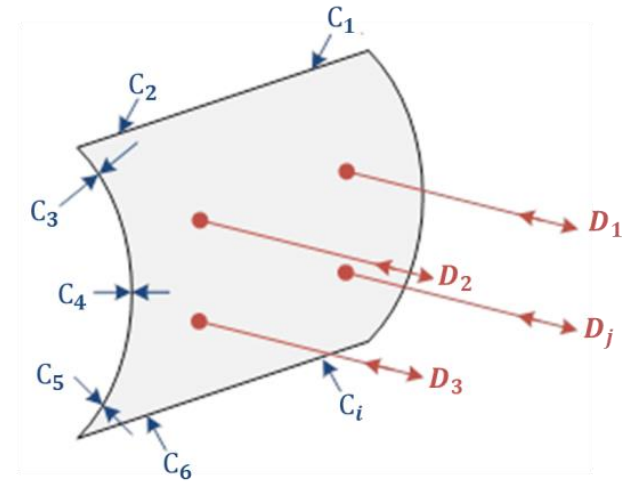

Fig. 1. Problem description

Describing the behavior of the assembly as a multi-input / multi-output linear function depending on the displacement of the positioning contact points, the component characteristics and the assembly process defined, we describe:

$$
\delta C_{i}=f\left(\delta D_{j}\right)
$$

Given the characteristics initial state $C_{i, \text { init }}$, the best fit problem is defined as:

$$
\text { to find } \delta D_{j} \text { so that } C_{i, \text { init }}-\delta C_{i} \in\left[C_{i, \min }, C_{i, \max }\right] \quad \forall i=(1, \infty)
$$

The function $f$ is defined by the sensitivity matrix or behavior model, described next point.

\subsection{Sensitivity matrix}

The sensitivity matrix or behavior model of the part can be generated following different methods (i.e. empirical, mathematical). In our case, we reused the Finite Element Model (FEM) created by Airbus Stress Department, used for full aircraft flight simulation during the aircraft design phase.

This FEM mesh needs to be split into section or component meshes, to keep only the corresponding part of the aircraft belonging to the case of study. As it was designed to analyze large in-flight loads and displacements, one task of the project will be validating its behavior on small displacements, which will be the case during the part assembly positioning.

On a second step, the mesh needs to be updated to the exact assembly condition of the assembly process stage of the case study. This means that the reused mesh might not be compliant to the real part status on assembly process stage under study.

The system characteristics (i.e. degrees of freedom) are defined as points in the part CAD model, kinematic links of the jig to the part, requirements on the part and degrees of freedom in each point. These points are transformed to the corresponding 
nodes of the FEM model, to extract a reduced sensitivity matrix with only the points of interest.

This CAD-CAE link can be done through different process (i.e. point to closest node or point to set of neighbor nodes) [5] [13] [14]. On this project we made the link by point to closest node method. Further hypothesis that could impact the model are, the manufactured composite material deviation, geometrical variations, jigs knowledge on operation direction and degrees of freedom, among others.

\subsection{Process}

Once the sensitivity matrix is generated on an off-line process as described in the previous point, the following on-line process would be followed at the shopfloor for each Manufacturing Serial Number (MSN) or individual aircraft:

1. Load assembly definition and sensitivity matrix ( $\mathrm{K}$ on equation 5 ) of the case of use in FITFLEX software interface.

2. Get from the measurement system (i.e. laser tracker) and jig force sensors in the kinematic links, the initial state of the part position and forces, and import the values $\left(C_{i}\right.$ and $\mathrm{F}$ on equation 5$)$.

3. Execute the optimization mathematic algorithm, solving the matrix system to obtain $D_{j}$ :

$$
[K]\left[\begin{array}{l}
C_{i} \\
D_{j}
\end{array}\right]=\left[\begin{array}{l}
0 \\
F
\end{array}\right]
$$

making as first step a function minimization without constrains and a second step considering the tolerance domains.

A so called virtual test is also possible to generate, simulating the result of the part constrains and requirements when making a set of displacements in the jig actuators.

\section{Use case}

The case subject of study on FITFLEX project is the A350 XWB rear fuselage Section 16 assembly, carried out at Airbus facilities in Hamburg, Germany. This assembly process comprises four shells and one grid floor; all made from carbon fiber reinforced plastic material (CFRP). Figure 2 shows the five components during assembly process, conforming section 16 . The project was focused only in the left side shell positioning process.

The CFRP material has stronger restrictions on gaps at interfaces. This is the reason why a specific jig was designed for this parts positioning, giving the possibility to adapt the form of the part by introducing small constrains. 


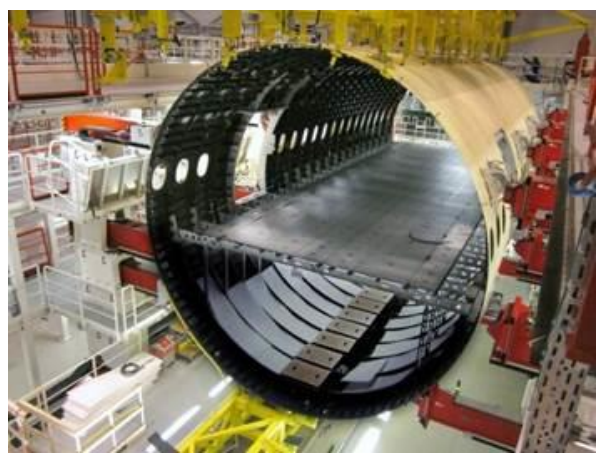

Fig. 2. Image of Airbus A350 XWB rear fuselage Section 16 at the assembly station

The Left Side Shell (LSS) have approximate 14 meters long and 5 meters of cord on the frontal frame, and it is positioned at the assembly station by 12 hoisting points, that can generate a rigid body translation and rotation to the part or that can be independently activated, moving each actuator at a time on a flexible movement. These hoisting points are the positioning control points of our problem definition, as shown on the figure 3. The requirement points are position characteristics, in the points of the part contour and along selected frames, and force characteristics, at the actuators location.

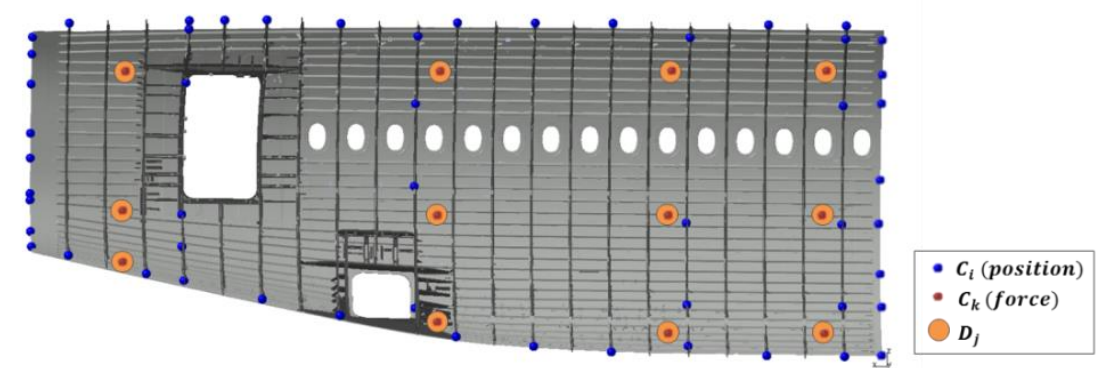

Fig. 3. Variables defined for the Left side shell case study

The system characteristics (i.e. degrees of freedom) are defined as points in the $\mathrm{CAD}$, mapped to the corresponding nodes of the FEM model, to extract a reduced stiffness matrix, behavior model of our system. This process is made only one time on a preparation phase, and for this project it was made using ANATOLE and ABAQUS tools [5].

On the shop floor, for each MSN Left Side Shell positioning process, FITFLEX software will be executed loading the assembly definition and behavior model on a new project. Figure 4 shows the work flow of the software interface, being the first image the new project generated.

After measuring the initial forces and initial position of the part at the assembly station, the values are imported using an excel template file, showing in the software interface the state of compliance of the requirements using a color code, which can be seen on Figure 4 second image. Then, an optimization function should be executed, to 
obtaining the rigid body move and flexible move that should be applied on the jig actuators to get a LSS position compliant to the requirements, shown on Figure 4 third image.

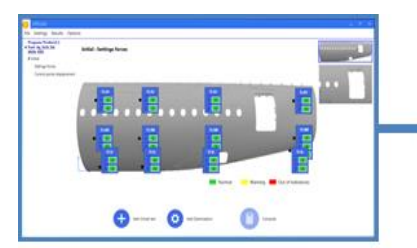

(1)

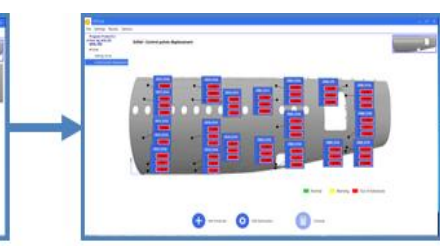

(2)

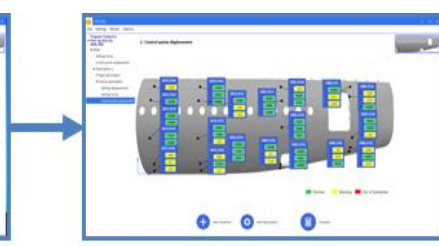

(3)

Fig. 4. FITFLEX software workflow

\section{$5 \quad$ Results and Discussion}

The use case objective was to introduce a behavior model that could help to find more quickly a convenient situation for the shell according to gaps specifications and force limitation on actuators. As presented in the previous chapter, our approach is based on a FEM model and on the capture of an initial state with force and dimensional data. The validation of FITFLEX approach on the use case is proposed through an overall process validation and justifications based on elementary tests.

\subsection{Process validation}

Using FITFLEX solution with the appropriate model loaded, the following validation has been done:

1. Introduce a LSS panel on the jig (without any specific conditions)

2. Measure target points position and force in actuators (to obtain initial state)

3. By using FIFLEX:

- Introduce values of initial state

- Get an overall status on gaps and force situation to targets

- Run optimization

- Identify rigid movement + smaller flexible displacement of actuators

- Get an overall status of final state where all requirements are met (Gap and force).

4. Execute displacement on actuators (rigid + flexible).

This process with FITFLEX is almost immediate compared to current iterative and manual setting process. However, even if the predicted final situation deviates from the final real situation in meeting all requirements, a second FITFLEX optimization can be made using the first setting result as a new initial state, leading to an acceptable situation. 


\subsection{Part geometrical variability}

A part geometrical deviation from its nominal geometry can be caused by several reasons (i.e. manufacturing process). A geometrical variability analysis was made on the LSS, to assess an order of magnitude of the part variability, as well as a process quality indicator for the behavior model to comply with, using historical measurements of several MSN.

\subsection{MSN experiences versus. behavior model simulations}

Two Measurement Campaigns were carried out in different MSN, to analyze the real LSS behavior on interface edge points. The complete set of points were measured during 12 load cases (activating each one of the 12 jig actuators for each load case), recording the actuators displacements and initial-final force.

The load cases were simulated with several boundary condition options, and the results were compared to the measurement campaign experience, analyzing:

- the FEM model used from Airbus Design Office (definition, usability and simplification)

- Simulation vs MSN global results, identifying a correct behavior (within the quality indicator given by the LSS geometrical variability) and correct definition of simulation conditions.

In a real MSN positioning process of the LSS, after the LSS is located in datum position on the station, the initial state of the position requirements have deviations in the order of centimeters on all directions. From this initial state, the displacements that have to be applied on each actuator are also in the order of magnitude of centimeters in the corresponding actuator degree of freedom. In this initial state, after simulating the displacements applied on the actuators, the standard deviation between the experiences versus simulation results is in the order of millimeters.

On following loops for the real MSN positioning, the requirements deviations are smaller (order of millimeters). Simulating the real displacements made on these loops (also in the order of millimeters), the experiences versus simulation standard deviation is lower than half of a millimeter.

The prediction model shows therefore consistent results for all tested MSN, meaning also this model is not a dedicated model for one MSN. The simulation results shown a force deviation compared to the experiences, and possible causes are under investigation: the decimal numbers considered on linear equation solver, the point where the force is measured vs. point defined on the simulation, and elasticity on the kinematic links.

\section{$5.3 \quad$ Optimizations}

Analyzing the result of these simulations, and in order to maintain the problem within the small displacement assumption and system linearity, to calculate the optimum actuator displacements, we incorporated as first step a rigid body move, to min- 
imize the deviations without introducing any constrains, and as second step, use a flexible body move best fit.

Several optimization scenarios can be generated varying weight on overall gaps (requirements), overall forces, gaps on each point independently (i.e. to give priority to interface points), or force on each actuator independently. An optimization final test in the assembly station was not possible due to ramp-up constrains, but optimization show promising results.

\section{Conclusion}

In this paper, a new approach for a flexible best fit positioning of parts to respect defined functional requirements, was presented. Its originality resides on the re-use of a physical model description made during aircraft design phase to adjust a real part during its assembly, considering a system linear behavior within small displacements.

The tool developed for shop floor usage need no FEA computation, solving an optimization problem in short time to find the best part positioning, that fulfills the requirements while controls and minimizes the constrains introduced to the part.

The new solution was applied to a use case (left side shell of A350 XWB rear fuselage) to evaluate the developments and their implementation under industrial conditions. Different aircrafts historical records were used to test and validate the developed applications, the prediction model shown consistent results for all tested MSN and optimization simulations shown promising results. Although the results were correct and demonstrated the suitability of the developments, additional testing of optimization algorithms on real part positioning is needed to validate full capabilities.

When compare to the current approach, the new flexible best fit could conduct to an increase on control of constrains introduced to the part, reduced positioning time for assembly, and creates new possibilities for new assembly process with flexible jigs usage to comply with requirements while controlling constrains.

A future work is to test and adapt the developed applications to a multi-part positioning problem. This is a different approach to the one used in this work, as it would consider overall assembly deviations and tolerance zones, to achieve the full part compliance to the ultimate requirement of the assembled part.

\section{$7 \quad$ Acknowledgment}

FITFLEX project was partially funded by LOCOMACHS EU project. Authors want to thank to their colleagues from Airbus Hamburg, IMACS and DPS for their contributions and kind collaboration. 


\section{$8 \quad$ References}

[1] A. Gomez, V. Olmos, J. Racero, J. Rios, R. Arista, F. Mas, Development based on reverse engineering to manufacture aircraft custom-made parts. International Journal of Mechatronics and Manufacturing Systems. Vol. 10 (1), pp 40-58. 2017.

[2] N. Chevassus et al., A New Approach for Best Fit Assembly Based on the Behavior of Components. SAE Technical Paper. 2006.

[3] M. Mounaud, F. Thiébaut, P. Bourdet, H. Falgarone, N. Chevassus. Assembly Sequence Influence on Geometric Deviations of Compliant Parts. International Journal of Production Research Vol. 49(4). 2011.

[4] LOCOMACHS: LOw COst Manufacturing and Assembly of Composite and Hybrid Structures. http://www.locomachs.eu/ (last accesses May 2017)

[5] H. Falgarone, F. Thiébaut, J. Coloos, L. Mathieu. Variation simulation during assembly of non-rigid components. Realistic assembly simulation with ANATOLEFLEX software. 14th CIRP CAT 2016 - CIRP Conference on Computer Aided Tolerancing. 2016.

[6] L. Andolfatto. Assistance à l'élaboration de gammes d'assemblage innovantes de structures composites. Autre. École normale supérieure de Cachan - ENS Cachan, 2013

[7] C. Lacroix. Caractérisation géométrique des assemblages flexibles par la mesure. Autre. École normale supérieure de Cachan - ENS Cachan, 2015. Français.

[8] Liu, G., Huan, H., \& Ke, Y. (2014). Study on analysis and prediction of riveting assembly variation of aircraft fuselage panel. The International Journal of Advanced Manufacturing Technology, 75(5-8), 991-1003.

[9] S. Ghandi, E. Masehian. Assembly sequence planning of rigid and flexible parts. Journal of Manufacturing Systems. Vol. 36, pp128-146. 2015.

[10] D.Gouyou, Y. Ledoux, D. Teissandier et al. Tolerance analysis of overconstrained and flexible assemblies by polytopes and finite element computations: application to a flange Research in Engineering Design. 2017..

[11] Cheng, H., Li, Y., Zhang, K. F., Mu, W. Q., \& Liu, B. F. (2011). Variation modeling of aeronautical thin-walled structures with multi-state riveting.

Journal of Manufacturing Systems, 30(2), 101-115.

[12] Liao, X., \& Wang, G. G. (2008). Simultaneous optimization of fixture and joint positions for non-rigid sheet metal assembly. The International Journal of Advanced Manufacturing Technology, 36(3-4), 386-394.

[13] E. Paroissien, M. Sartor, J. Huet, F. Lachaud. Hybrid (Bolted/Bonded) Joints Applied to Aeronautic Parts: Analytical Two-Dimensional Model of a Single-Lap Joint. Journal of Aircraft. Vol. 4 (2), pp 573-582. 2007.

[14] A.P. Gonze, J. Verstuyft. Associative Sizing of Aeronautical Structures from Catia V5 to Samcef: Applications to Static and Bird Impact Analyses. 9th SAMTECH Users Conference, 2005 